\title{
Patterns of Care and Predictors of Survival among DCIS Patients: An NCDB Analysis
}

\author{
Justine S Broecker ${ }^{*}$, Yuan S Liu' ${ }^{2}$, Betsey Dewey ${ }^{3}$, Toncred Styblo ${ }^{4}$ and Theresa S Gillespie \\ ${ }^{1}$ Department of Surgery, Mayo Clinic Florida, USA \\ ${ }^{2}$ Department of Biostatics, Emory University School of Medicine, USA \\ ${ }^{3}$ Emory School of Public Health, USA \\ ${ }^{4}$ Department of Surgical Oncology, Winship Cancer Institute, Emory University School of \\ Medicine, USA
}

\begin{abstract}
Introduction: The optimal treatment of ductal carcinoma in situ (DCIS) remains controversial. The aims of this study were to: 1) Evaluate patterns of treatment and, 2) Identify predictors of survival among patients diagnosed with DCIS.

Methods: The National Cancer Database (NCDB) was queried to identify all patients diagnosed with DCIS between 20042015. After applying exclusion criteria, Cox proportional hazards and Kaplan-Meier analysis were performed to compare treatment groups and estimate risk of death stratified by demographics, clinical features, and treatment delivered. An average treatment effect (ATE) was calculated between three matched treatment cohorts of interest: Lumpectomy alone, extended local therapy (lumpectomy/radiation or mastectomy) and extended local therapy + anti-hormonal therapy.

Results: Among 34,444 patients diagnosed with DCIS who met inclusion criteria, the mean age at diagnosis was 60 . Patients who received lumpectomy alone were older, and had smaller, and more well-differentiated tumors compared to other treatment cohorts $(p<0.001)$. After calculating ATE among matched cohorts, patients who underwent extended local therapy ( $\mathrm{HR}=0.81,95 \% \mathrm{Cl}: 0.73-0.90)$ and extended local therapy + anti-hormonal therapy $(\mathrm{HR}=0.61,95 \% \mathrm{Cl}: 0.54-$ 0.68 ) had improved survival compared to lumpectomy alone $(p<0.001)$. At 120 months, anti-hormonal therapy had a significant impact upon survival for ER/PR positive tumors ( $H R=1.45, p<0.001)$ but not ER/PR negative tumors $(H R=$ $1.15, p=0.188)$. Additional predictors of reduced survival on MVA included African-American race $(\mathrm{HR}=1.37,95 \% \mathrm{Cl}$ : 1.21-1.55), increased Charlson-Deyo score ( $\mathrm{HR}=2.82,95 \% \mathrm{Cl}: 2.37-3.36)$, older age at diagnosis ( $\mathrm{HR}=6.4895 \% \mathrm{Cl}: 5.31-$ 7.91), and Medicaid insurance ( $\mathrm{HR}=2.03,95 \% \mathrm{Cl}: 1.64-2.52)$ (all $\mathrm{p}<0.001)$.

Conclusions: Among patients diagnosed with DCIS, extended local therapy plus the addition of anti-hormonal therapy significantly reduced mortality by $2.2 \%$ and $3.5 \%$ at 60 months among matched cohorts. Although the NCDB does not capture recurrence or breast-cancer specific mortality, these results suggest that additional therapy beyond surgery alone may improve mortality for patients diagnosed with DCIS and warrants further investigation.
\end{abstract}

\section{Abbreviations}

DCIS: Ductal Carcinoma in Situ; NCCN: National Comprehensive Cancer Network; RT: Radiation Therapy; NCDB: National Cancer Database; PUF: Participant User Data File; COC: Commission on Cancer; ATE: Average Treatment Effect; Cl: Confidence Intervals

\section{Introduction}

The treatment of ductal carcinoma in situ (DCIS, intraductal carcinoma, stage " 0 " breast cancer) of the breast is varied and controversial, with overtreatment and under-treatment both causes of concern [1-7]. Reflecting the lack of consensus, national guidelines allow for a wide range of local treatment options [8-11]. Current National Comprehensive Cancer Network (NCCN) guidelines recommend complete local therapy (lumpectomy with radiation) with the addition of anti-hormonal therapy as appropriate, but also consider the omission of radiation therapy (RT) and/or anti-hormonal therapy and use of mastectomy appropriate for certain patient populations. Radiation and anti-hormonal therapy have

* Corresponding author: Justine S Broecker, Department of Surgery, Mayo Clinic Florida, Jacksonville, Florida, USA

Accepted: September 29, 2020

Published online: October 01, 2020

Citation: Broecker JS, Liu YS, Dewey B, et al. (2020) Patterns of Care and Predictors of Survival among DCIS Patients: An NCDB Analysis. Ann Breast Cancer Ther 4(1):58-68 
been shown to reduce recurrence without affecting survival, and outcomes of surveillance without surgery are being evaluated by several open randomized clinical trials [12-15]. The aim of this study was to examine patterns of care among patients captured by the National Cancer Database (NCDB) diagnosed with DCIS and to compare clinic opathologic features and overall survival (OS) among varied treatment cohorts. We chose three treatment cohorts of particular interest as follows: 1) Lumpectomy alone, 2) Extended local therapy (lumpectomy + XRT or mastectomy), and 3) Extended local therapy plus anti-hormonal therapy.

\section{Methods}

\section{Data source}

Our data sample was extracted from the National Cancer Database (NCDB)'s Participant User Data File (PUF) for breast cancer. Created in 1989 by the American College of Surgeons' Commission on Cancer ( $\mathrm{CoC}$ ) and the American Cancer Society, the NCDB is a registry that captures roughly $70 \%$ of U.S. cancer cases The NCDB contains approximately 34 million records from $\mathrm{CoC}$ accredited cancer registries across the United States. The registry includes demographics, clinic pathologic and treatment data, OS is measured but not recurrence or cancer-specific survival [16]. The NCDB PUF contains de-identified patient and facility data and therefore, is complaint with the Health Insurance Portability and Accountability Act (HIPAA) and exempt from Institutional Review Board review.

\section{Study population}

Our study population consisted of all NCDB reported cases of DCIS diagnosed between 2004 and 2015. We included stage 0 breast cancers and first or only cancers. Histologic patterns included were intraductal carcinoma, comedocarcinoma, intraductal papillary adenocarcinoma, and intraductal concomitant with lobular carcinoma, all non-infiltrating. We excluded male patients, invasive and microinvasive tumors, lobular carcinoma in situ without associated DCIS, positive nodes, positive margins, missing anti-hormonal receptor statuses, cases where treatment was not performed at the reporting facility, cases without surgical excision of the primary tumor site, all 90-day mortalities, missing outcomes or undetermined treatment patterns. Margin size is not captured by the NCDB. Since our interest was to compare local and systemic therapy groups, patients who had bilateral mastectomy were excluded as this additional surgery was beyond standard, recommended local control. Three treatment cohorts of interest were then defined as follows: 1) Lumpectomy alone, 2) Extended local therapy (lumpectomy with RT or mastectomy), 3) Extended local therapy with anti-hormonal therapy.

\section{Statistical methods}

The frequencies of variables of interest among the entire study cohort are listed in (Table 1). Univariate (UVA) and multivariable (MVA) analysis (built by backward variable se-

Table 1: Characteristics of patients diagnosed with DCIS.

\begin{tabular}{|c|c|c|}
\hline \multicolumn{2}{|r|}{ Variable } & $n(\%)=34,444$ \\
\hline \multirow[t]{3}{*}{ Treatment Cohort } & Lumpectomy Alone & $4,768(13.8)$ \\
\hline & Local Therapy & $15,446(44.8)$ \\
\hline & Local therapy + Hormone & $14,230(41.3)$ \\
\hline \multirow[t]{4}{*}{ Age (quartile) } & $>=23,<=51$ & $9,428(27.4)$ \\
\hline & $>51,<=59$ & $8,059(23.4)$ \\
\hline & $>59,<=68$ & $8,894(25.8)$ \\
\hline & $>68,<=90$ & $8,063(23.4)$ \\
\hline \multirow[t]{3}{*}{ Race } & White & $28,384(82.4)$ \\
\hline & Black & 4,249 (12.3) \\
\hline & Other/Unknown & $1,811(5.3)$ \\
\hline \multirow[t]{3}{*}{ Charlson-Deyo Score } & 0 & $29.747(86.4)$ \\
\hline & 1 & $3,975(12.3)$ \\
\hline & $2+$ & $722(2.1)$ \\
\hline \multirow[t]{5}{*}{ Facility Type } & Community Cancer Program/Other & $3,171(9.4)$ \\
\hline & Comprehensive Community Cancer Program & $16,961(50.3)$ \\
\hline & Academic/Research Program & $9,043(26.8)$ \\
\hline & Integrated Network Cancer Program & $4,544(13.5)$ \\
\hline & Missing & 725 \\
\hline \multirow[t]{4}{*}{ Primary Payor } & Not Insured/Unknown & $914(2.7)$ \\
\hline & Private & $20.757(60.3)$ \\
\hline & Medicaid/Other Government & $1,822(5.3)$ \\
\hline & Medicare & 10,951 (31.8) \\
\hline
\end{tabular}


Citation: Broecker JS, Liu YS, Dewey B, et al. (2020) Patterns of Care and Predictors of Survival among DCIS Patients: An NCDB Analysis. Ann Breast Cancer Ther 4(1):58-68

\begin{tabular}{|c|c|c|}
\hline \multirow[t]{4}{*}{ Year of Dx } & $>=2004,<=2008$ & $11,027(32.0)$ \\
\hline & $>2008,<=2010$ & $11,320(32.9)$ \\
\hline & $>2010,<=2012$ & $6,021(17.5)$ \\
\hline & $>2012,<=2013$ & $6,076(17.6)$ \\
\hline \multirow[t]{4}{*}{ Histology } & Ductal & $25,358(73.6)$ \\
\hline & Comedocarcinoma & $6,130(17.8)$ \\
\hline & Papillary & $1,082(3.1)$ \\
\hline & Ductal + lobular & $1,874(5.4)$ \\
\hline \multirow[t]{2}{*}{ Hormonal Status } & $E R / P R+$ & $5,783(16.8)$ \\
\hline & ER/PR - & $28,661(83.2)$ \\
\hline \multirow[t]{3}{*}{ HER-2 Status } & Negative & $2317(6.7)$ \\
\hline & Positive & $1258(3.7)$ \\
\hline & Unknown & $30,869(89.6)$ \\
\hline \multirow[t]{4}{*}{ Grade } & Well Differentiated & $3,497(10.2)$ \\
\hline & Moderately Differentiated & $9,870(28.7)$ \\
\hline & Poorly Differentiated/Undifferentiated & $14,005(40.7)$ \\
\hline & Cell Type Not Determined & $7.072(20.5)$ \\
\hline \multirow[t]{5}{*}{ Tumor Size (quartile) (cm) } & $>=0.1,<=0.5$ & $7,144(20.7)$ \\
\hline & $>0.5,<=1$ & 5,957 (17.3) \\
\hline & $>1,<=1.9$ & $5,695(16.5)$ \\
\hline & $>1.9,<=98.8$ & $6,218(18.1)$ \\
\hline & Unknown & $9,430(27.4)$ \\
\hline \multirow[t]{2}{*}{ Hormonal Therapy } & No & $20,214(58.7)$ \\
\hline & Yes & $14,230(41.3)$ \\
\hline \multirow[t]{3}{*}{ Immunotherapy } & No & 34,293 (99.6) \\
\hline & Yes & $60(0.2)$ \\
\hline & Unknown & $91(0.3)$ \\
\hline \multirow[t]{2}{*}{ Radiation } & No & $11,900(34.5)$ \\
\hline & Yes & $22,544(65.5)$ \\
\hline
\end{tabular}

"Local Therapy $=$ Lumpectomy + Radiation

Table 2: Univariate and Multivariate Model for the Association with Overall Survival (OS).

\begin{tabular}{|c|c|c|c|c|c|}
\hline \multicolumn{2}{|l|}{ Variable } & $\begin{array}{l}\text { UVA HR } \\
(95 \% \mathrm{Cl})\end{array}$ & p-value & $\begin{array}{l}\text { MVA HR } \\
(95 \% \mathrm{Cl})\end{array}$ & p-value \\
\hline \multirow[t]{3}{*}{ Treatment Cohort } & Local Therapy & $0.57(0.51-0.63)$ & \multirow[t]{3}{*}{$<0.001$} & $0.69(0.62-0.77)$ & \multirow[t]{3}{*}{$<0.001$} \\
\hline & Local Therapy + Hormone & $0.33(0.30-0.38)$ & & $0.51(0.45-0.58)$ & \\
\hline & Lumpectomy Alone & Ref & & Ref & \\
\hline \multirow[t]{4}{*}{ Age (quartile) } & $>68,<=90$ & $11.64(9.89-13.70)$ & \multirow[t]{4}{*}{$<0.001$} & $6.48(5.31-7.91)$ & \multirow[t]{4}{*}{$<0.001$} \\
\hline & $>59,<=68$ & $3.28(2.74-3.93)$ & & $2.49(2.05-3.01)$ & \\
\hline & $>51,<=59$ & $1.77(1.45-2.17)$ & & $1.67(1.36-2.05)$ & \\
\hline & $>=23,<=51$ & Ref & & Ref & \\
\hline \multirow[t]{3}{*}{ Race } & Black & $1.31(1.16-1.48)$ & \multirow[t]{3}{*}{$<0.001$} & $1.37(1.21-1.55)$ & \multirow[t]{3}{*}{$<0.001$} \\
\hline & Other/Unknown & $0.51(0.40-0.67)$ & & $0.67(0.52-0.88)$ & \\
\hline & White & Ref & & Ref & \\
\hline
\end{tabular}


Citation: Broecker JS, Liu YS, Dewey B, et al. (2020) Patterns of Care and Predictors of Survival among DCIS Patients: An NCDB Analysis. Ann Breast Cancer Ther 4(1):58-68

\begin{tabular}{|c|c|c|c|c|c|}
\hline \multirow{3}{*}{ Charlson-Deyo Score } & $2+$ & $4.58(3.85-5.45)$ & \multirow[t]{3}{*}{$<0.001$} & $2.82(2.37-3.36)$ & \multirow[t]{3}{*}{$<0.001$} \\
\hline & 1 & $1.93(1.72-2.16)$ & & $1.42(1.27-1.59)$ & \\
\hline & 0 & Ref & & Ref & \\
\hline \multirow{4}{*}{ Facility Type } & Community Cancer Program/Other & $1.32(1.13-1.53)$ & \multirow[t]{4}{*}{0.006} & $1.21(1.04-1.41)$ & \multirow[t]{4}{*}{0.107} \\
\hline & $\begin{array}{l}\text { Comprehensive Community Cancer } \\
\text { Program }\end{array}$ & $1.09(0.98-1.20)$ & & $1.03(0.93-1.15)$ & \\
\hline & Integrated Network Cancer Program & $1.06(0.92-1.23)$ & & $1.04(0.90-1.21)$ & \\
\hline & Academic/Research Program & Ref & & Ref & \\
\hline \multirow{4}{*}{ Primary Payor } & Not Insured/Unknown & $2.48(1.89-3.26)$ & \multirow[t]{4}{*}{$<0.001$} & $1.87(1.42-2.48)$ & \multirow[t]{4}{*}{$<0.001$} \\
\hline & Medicaid/Other Government & $2.35(1.91-2.91)$ & & $2.03(1.64-2.52)$ & \\
\hline & Medicare & $5.42(4.92-5.97)$ & & $1.74(1.53-1.98)$ & \\
\hline & Private & Ref & & Ref & \\
\hline \multirow[t]{4}{*}{ Year of Diagnosis } & $>=2004,<=2008$ & $0.93(0.78-1.11)$ & \multirow[t]{4}{*}{0.204} & \multirow[t]{4}{*}{ NS } & \\
\hline & $>2008,<=2010$ & $1.04(0.87-1.24)$ & & & \\
\hline & $>2010,<=2012$ & $0.96(0.78-1.18)$ & & & \\
\hline & $>2012,<=2013$ & Ref & & & \\
\hline \multirow[t]{4}{*}{ Histology } & Comedocarcinoma & $1.04(0.93-1.16)$ & \multirow[t]{4}{*}{$<0.001$} & \multirow[t]{4}{*}{ NS } & \\
\hline & Papillary & $1.56(1.27-1.90)$ & & & \\
\hline & Ductal + lobular & $0.81(0.66-1.00)$ & & & \\
\hline & Ductal & Ref & & & \\
\hline \multirow[t]{2}{*}{ ERPR } & ERPR+ & $0.76(0.69-0.85)$ & \multirow[t]{2}{*}{$<0.001$} & $0.95(0.85-1.05)$ & \multirow[t]{2}{*}{0.348} \\
\hline & ERPR - & Ref & & Ref & \\
\hline \multirow[t]{3}{*}{ HER2 } & Negative & $1.08(0.78-1.48)$ & \multirow[t]{3}{*}{0.582} & \multirow[t]{3}{*}{ NS } & \\
\hline & Unknown & $0.97(0.75-1.27)$ & & & \\
\hline & Positive & Ref & & & \\
\hline \multirow[t]{4}{*}{ Grade } & Moderately Differentiated & $1.0(0.87-1.17)$ & \multirow[t]{4}{*}{0.765} & \multirow[t]{4}{*}{ NS } & \\
\hline & Poorly Differentiated/Undifferentiated & $0.96(0.83-1.10)$ & & & \\
\hline & Cell Type Not Determined & $1.00(0.85-1.17)$ & & & \\
\hline & Well differentiated & Ref & & & \\
\hline Tumor Size Quartile & $>0.5,<1$ & & 0.002 & $1.25(1.09-1.44)$ & 0.004 \\
\hline & $>1,<=2$ & $1.28(1.12-1.47)$ & & $1.17(1.02-1.36)$ & \\
\hline & $>2,<=99$ & $1.19(1.03-1.37)$ & & $1.27(1.10-1.46)$ & \\
\hline & Unknown & $1.26(1.10-1.45)$ & & $1.11(0.98-1.26)$ & \\
\hline & $>0.1,<0.5$ & Ref & & Ref & \\
\hline
\end{tabular}

*NS: Not selected by the backward variable elimination, and not significant at $p<0.2$.

lection with alpha $=0.2$ removal criteria) were performed to determine associations between variables of interest and OS using a Cox proportional hazard model (yielding hazard ratios [HR] with $95 \%$ confidence intervals $[\mathrm{Cl}]$ ) as reported in (Table 2). Ten-year OS using Kaplan Meier analysis was conducted for treatment cohorts of interest (with log rank test performed to determine $p$ values) (Figure 1). Associations between variables of interest and these three treatment groups were examined using chi-square for categorical variables and ANOVA for continuous covariates (Table 3). To further reduce the selection bias, the inverse probability treatment weighting method, a propensity score based approach was also implemented to balance patient's baseline characteristics. A multinomial logistic regression model was carried out to estimate the probabilities that a patient would receive either lumpectomy, extended local therapy, or extended local therapy + anti-hormonal therapy based on their baseline covariates that also predict overall survival. The balance of covariate between cohorts was evaluated by the standardized differences and a value of $<0.1$ was considered as negligible imbalance [17]. The average treatment effect (ATE) by the three cohorts associated with OS was estimated in a weighted Cox proportional hazard model. The subgroup analyses were carried out in the multivariable model with interaction between treatment groups and ER/PR status. The analyses were done in SAS 9.4 and Winship BBISR SAS macros, and significance level was set at alpha $<0.05$ [18]. 
Citation: Broecker JS, Liu YS, Dewey B, et al. (2020) Patterns of Care and Predictors of Survival among DCIS Patients: An NCDB Analysis. Ann Breast Cancer Ther 4(1):58-68

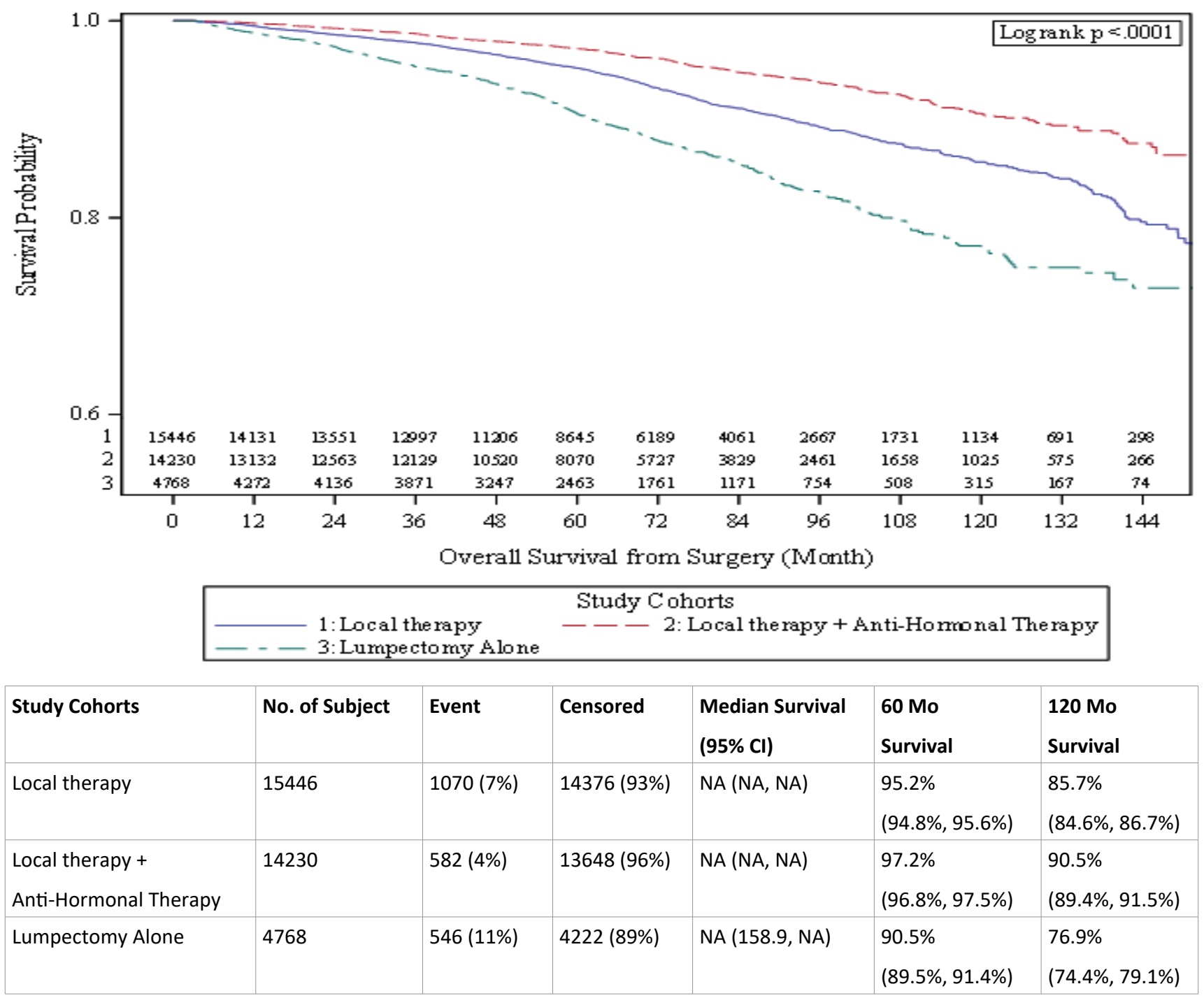

Figure 1: Overall Survival all patients diagnosed with DCIS stratified by treatment group.

Table 3: Association between variables of interest and three treatment groups.

\begin{tabular}{|c|c|c|c|c|c|}
\hline \multicolumn{2}{|l|}{ Variable } & $\begin{array}{l}\text { Lumpectomy Alone } \\
\qquad n=4768\end{array}$ & $\begin{array}{l}\text { Local Therapy } \\
\qquad n=15446\end{array}$ & $\begin{array}{l}\text { Systemic Therapy } \\
\qquad n=14230\end{array}$ & p-value \\
\hline \multirow[t]{3}{*}{ Age at diagnosis } & Mean & 63.7 & 60.03 & 57.8 & \multirow[t]{3}{*}{$<0.001$} \\
\hline & Median & 64 & 60 & 58 & \\
\hline & Std Dev & 13.25 & 11.77 & 10.35 & \\
\hline \multirow[t]{4}{*}{ Age (quartile) } & $>=23,<=51$ & $1003(21.04)$ & $4083(26.43)$ & $4342(30.51)$ & \multirow[t]{4}{*}{$<0.001$} \\
\hline & $>51<=59$ & $880(18.46)$ & 3456 (22.37) & 3723 (26.16) & \\
\hline & $>59,<=68$ & 1045 (21.92) & $3986(25.81)$ & $3863(27.15)$ & \\
\hline & $>68,<=90$ & 1840 (38.59) & 3921 (25.39) & $2302(16.18)$ & \\
\hline \multirow[t]{3}{*}{ Race } & White & $3930(82.42)$ & $12852(83.21)$ & $11602(81.53)$ & \multirow[t]{3}{*}{0.002} \\
\hline & Black & $576(12.08)$ & 1804 (11.68) & 1869 (13.13) & \\
\hline & Other/Unknown & 262 (5.49) & $790(5.11)$ & 759 (5.33) & \\
\hline \multirow[t]{3}{*}{ Carlson-Deyo Score } & 0 & 4132 (86.66) & $13197(85.44)$ & $12418(87.27)$ & \multirow[t]{3}{*}{$<0.001$} \\
\hline & 1 & $523(10.97)$ & $1901(12.31)$ & 1551 (10.9) & \\
\hline & $2+$ & $113(2.37)$ & $348(2.25)$ & $261(1.83)$ & \\
\hline
\end{tabular}


Citation: Broecker JS, Liu YS, Dewey B, et al. (2020) Patterns of Care and Predictors of Survival among DCIS Patients: An NCDB Analysis. Ann Breast Cancer Ther 4(1):58-68

\begin{tabular}{|c|c|c|c|c|c|}
\hline \multirow[t]{4}{*}{ Facility Type } & $\begin{array}{l}\text { Commun. Cancer Program/ } \\
\text { Other }\end{array}$ & $431(9.22)$ & $1234(8.2)$ & $1506(10.77)$ & \multirow[t]{4}{*}{$<0.001$} \\
\hline & $\begin{array}{l}\text { Comp Commun Cancer } \\
\text { Program }\end{array}$ & $2396(51.24)$ & 7559 (50.21) & 7006 (50.08) & \\
\hline & Academic/Research Program & $1228(51.24)$ & $4216(28.01)$ & 3599 (25.73) & \\
\hline & $\begin{array}{l}\text { Integrated Network Cancer } \\
\text { Program }\end{array}$ & $621(13.28)$ & $2045(13.58)$ & $1878(13.42)$ & \\
\hline \multirow[t]{4}{*}{ Primary Payor } & Not Insured/Unknown & $141(2.96)$ & $392(2.54)$ & $381(2.68)$ & \multirow[t]{4}{*}{$<0.001$} \\
\hline & Private & 2367 (49.64) & 9087 (58.83) & 9303 (65.38) & \\
\hline & Medicaid/Other Government & $208(4.36)$ & 739 (4.91) & $855(6.01)$ & \\
\hline & Medicare & 2052 (43.04) & $5208(33.72)$ & 3691 (25.94) & \\
\hline \multirow[t]{4}{*}{ Year of Diagnosis } & $>=2004,<=2008$ & $1603(33.62)$ & $5078(32.88)$ & $4346(30.54)$ & \multirow[t]{4}{*}{$<0.001$} \\
\hline & $>2008,<=2010$ & $1508(31.63)$ & 5199 (33.66) & $4613(32.42)$ & \\
\hline & $>2010,<=2012$ & $813(17.05)$ & 2609 (16.89) & 2599 (18.26) & \\
\hline & $>2012,<=2013$ & $844(17.7)$ & $2560(16.57)$ & $2672(18.78)$ & \\
\hline \multirow[t]{4}{*}{ Histology } & Ductal & 3696 (77.52) & $11163(72.27)$ & $10499(73.78)$ & \multirow[t]{4}{*}{$<0.001$} \\
\hline & Comedocarcinoma & $593(12.44)$ & $3108(20.12)$ & 2429 (17.07) & \\
\hline & Papillary & 238 (4.99) & $390(2.52)$ & 454 (3.19) & \\
\hline & Ductal + lobular & $241(5.05)$ & $785(5.08)$ & $848(5.96)$ & \\
\hline \multirow{2}{*}{$\begin{array}{l}\text { Hormonal Receptor } \\
\text { Status }\end{array}$} & ER/PR - & $723(15.16)$ & $4,648(30.09)$ & $412(2.9)$ & \multirow[t]{2}{*}{$<0.001$} \\
\hline & $E R / P R+$ & $4,045(84.84)$ & $10,798(69.91)$ & $13,818(97.1)$ & \\
\hline \multirow[t]{3}{*}{ HER2 } & Negative & $302(6.33)$ & $882(5.71)$ & $1,113(7.96)$ & \multirow[t]{3}{*}{$<0.001$} \\
\hline & Positive & $113(2.73)$ & $670(4.34)$ & $475(3.34)$ & \\
\hline & Unknown & 4,353 (91.3) & 13,894 (89.95) & $12,622(88.7)$ & \\
\hline \multirow[t]{4}{*}{ Grade } & Well Differentiated & $776(16.28)$ & $1202(7.78)$ & 1519 (10.67) & \multirow[t]{4}{*}{$<0.001$} \\
\hline & Moderately Differentiated & 1505 (31.56) & $3866(25.03)$ & 4499 (31.62) & \\
\hline & Poorly Differentiated & $1395(29.26)$ & 7247 (46.92) & 5363 (37.69) & \\
\hline & Cell Type Not Determined & $1092(22.9)$ & $3131(20.27)$ & $2849(20.02)$ & \\
\hline \multirow[t]{3}{*}{ Tumor Size (cm) } & Mean & 1.34 & 1.79 & 1.42 & \multirow[t]{3}{*}{$<0.001$} \\
\hline & Median & 0.8 & 1.2 & 1 & \\
\hline & Std. Dev & 3.48 & 2.89 & 2.32 & \\
\hline \multirow{5}{*}{$\begin{array}{l}\text { Tumor Size (quartile) } \\
(\mathrm{cm})\end{array}$} & $>=0.1,<=0.5$ & $1257(26.36)$ & $2790(18.06)$ & $3097(21.76)$ & \multirow[t]{5}{*}{$<0.001$} \\
\hline & $>0.5,<=1$ & $820(17.2)$ & $2537(16.42)$ & $2600(18.27)$ & \\
\hline & $>1,<=1.9$ & $660(13.84)$ & $2724(17.64)$ & $2311(16.24)$ & \\
\hline & $>1.9,<=9.8$ & 650 (13.63) & 3323 (2.51) & 2245 (15.78) & \\
\hline & Unknown & $1381(28.96)$ & 4072 (26.36) & 397 (27.95) & \\
\hline
\end{tabular}

\section{Results}

Study population, demographics, clinicopathologic and treatment variables

The NCDB breast PUF (2004-2014) identified a total of $2,696,734$ breast cancer cases. After applying the inclusion and exclusion criteria listed in (Table 4), a total of 34,444 patients met selection criteria with a median follow-up 63.9 months. Descriptive characteristics of this population regarding variables of interest are listed in (Table 1). The mean age of diagnosis was 59-years (std. dev. 12), the majority of patients were Caucasian (82.4\%), treated at a comprehensive community cancer program $(50.3 \%)$ and had private insur- ance $(60.3 \%)$. The treatment cohorts of interest are listed in (Table 1). The majority received additional treatment beyond lumpectomy: 4,768 (13.8\%) received lumpectomy alone $15,446(44.8 \%)$ received either lumpectomy with radiation or unilateral mastectomy 14,230 (41.3\%) received extended local therapy with additional anti-hormonal therapy.

Univariate, multivariate and Kaplan Meier analysis of variables associated with decreased overall survival and Kaplan Meier 10-year overall survival

The results of univariate (UVA) and multivariate (MVA) 
Citation: Broecker JS, Liu YS, Dewey B, et al. (2020) Patterns of Care and Predictors of Survival among DCIS Patients: An NCDB Analysis. Ann Breast Cancer Ther 4(1):58-68

Table 4: Inclusion and exclusion criteria

\begin{tabular}{|c|c|c|}
\hline Selection and Exclusion Criteria & Sample Size & Excluded \\
\hline NCDB Breast PUF Cancer Cases & 2696734 & - \\
\hline Year of diagnosis $2004 \sim 2015$ & 1982168 & 714566 \\
\hline Include sequence number in 0 or 1 & 1645418 & 336750 \\
\hline Exclude cases treatment decision was not done at reporting facility & 1581834 & 63584 \\
\hline Include Histology 8500850185038522 & 1176472 & 405362 \\
\hline Exclude male patients & 1165374 & 11098 \\
\hline Exclude tumor behavior as invasive & 157044 & 1008330 \\
\hline Include Clinical and Pathological stage 0 & 57329 & 99715 \\
\hline Include Diagnostic Confirmation as 12 & 57320 & 9 \\
\hline Exclude Tumor Invasive Component defined by CS_SITESPECIFIC_FACTOR_6 & 57297 & 23 \\
\hline Exclude cases with positive regional nodes & 57297 & 0 \\
\hline Exclude cases without surgery at primary site or unknown & 56988 & 309 \\
\hline Include Surgical Margin as negative & 54611 & 2377 \\
\hline Exclude cases died within 90 day after surgery & 54558 & 53 \\
\hline Exclude missing outcome & 53909 & 649 \\
\hline Include ERPR as 01 & 46641 & 7268 \\
\hline Exclude cases with bilateral mastectomy & 34444 & 12197 \\
\hline
\end{tabular}

*Sequence number: $0=$ only cancer, 1 = first cancer.

${ }^{*}$ Histology: $8500=$ DCIS, $8501=$ comedocarcinoma, non infiltrating, $8503=$ intraductal papillary adenocarcinoma, non infiltrating, $8522=$ DCIS + LCIS.

${ }^{*}$ CS_SITESPECIFIC_FACTOR_6 = Invasive Component.

Table 5: Multivariable model for OS in weighted overall samples and weighted subpopulation.

\begin{tabular}{|l|l|l|l|}
\hline Covariate & Level & Hazard Ration (95\% Cl) & HR p-value \\
\hline \multirow{3}{*}{ Overall } & Local therapy & $0.81(0.73-0.90)$ & $<0.001$ \\
\hline & Local therapy + Hormone & $0.61(0.54-0.68)$ & $<0.001$ \\
\hline & Lumpectomy Alone & Ref & \\
\hline Subgroup ${ }^{*}$ ER/PR+ & Local therapy vs. Lumpectomy Alone & $0.88(0.77-1.00)$ & 0.046 \\
\hline & Local therapy + Hormone vs. Lumpectomy Alone & $0.60(0.53-0.69)$ & $<0.001$ \\
\hline & Local therapy vs. Local therapy + Hormone & $1.45(1.30-1.62)$ & $<0.001$ \\
\hline Subgroup*: ER/PR- & Local therapy vs. Lumpectomy Alone & $0.61(0.48-0.78)$ & $<0.001$ \\
\hline & Local therapy + Hormone vs. Lumpectomy Alone & $0.53(0.41-0.68)$ & $<0.001$ \\
\cline { 2 - 3 } & Local therapy vs. Local therapy + Hormone & $1.15(0.93-1.42)$ & 0.188 \\
\hline
\end{tabular}

*The treatment comparison in the subgroups was estimated by the multivariable model with interaction, and the interaction $p$-value is 0.023 .

analysis of the association between variables of interest and OS are listed in (Table 2). Factors associated with decreased survival on univariate analysis included lumpectomy treatment alone, African-American race $(\mathrm{HR}=1.31,95 \% \mathrm{Cl}$ : 1.16 $1.48, \mathrm{p}<0.001)$, increased age $(\mathrm{HR}=11.64,95 \% \mathrm{Cl} 9.89-13.70$, $\mathrm{p}<0.001)$, increased Charlson-Deyo score $(\mathrm{HR}=4.58,95 \% \mathrm{Cl}$ : $3.85-5.45, p<0.001)$, any insurance other than private especially Medicare (HR $=5.42,95 \% \mathrm{Cl}: 4.92-5.97, \mathrm{p}<0.001)$, papillary histology (HR $=1.56,95 \% \mathrm{Cl}: 1.27-1.90, \mathrm{p}<0.001)$, ER/ $\mathrm{PR}$ negative receptor status $(\mathrm{HR}=0.76,95 \% \mathrm{Cl}: 0.69-0.85, \mathrm{p}$ $<0.001)$, and larger tumor size ( $\mathrm{HR}=1.26,95 \% \mathrm{Cl}: 1.10-1.45$, $\mathrm{p}<0.002)$. On MVA, lumpectomy alone, increased age ( $\mathrm{HR}=$ $6.48,95 \% \mathrm{Cl}: 5.31-7.91, \mathrm{p}<0.001)$, African-American race (HR
$=1.37,95 \% \mathrm{Cl}: 1.21-1.55, \mathrm{p}<0.001)$, increased Charlson-Deyo score $(\mathrm{HR}=2.82,95 \% \mathrm{Cl}: 2.37-3.36, \mathrm{p}<0.001)$, any insurance other than private especially Medicaid $(\mathrm{HR}=2.03,95 \% \mathrm{Cl}$ : $1.64-2.52, \mathrm{p}<0.001)$, and increased tumor size $(\mathrm{HR}=1.27$, $95 \% \mathrm{Cl}: 1.10-1.26, \mathrm{p}<0.004)$ were associated with decreased survival. Kaplan-Meier analysis was performed to determine ten-year OS and stratified by treatment cohorts of interest as shown in Figure 1. Lumpectomy alone had a decreased 10-year survival (76.9\%, 95\% Cl: 74.4-79.1\%) compared to extended local therapy $(85.7 \%, 95 \% \mathrm{Cl}: 84.6-86.7 \%)$ and extended local therapy with anti-hormonal therapy $(90.5 \%, 95 \% \mathrm{Cl}$ : 89.4-91.5\%) $(p<0.001)$. 
By utilizing inverse probability treatment weighting, a satisfactory covariate balance was achieved for the overall population as well as in the subgroups by ER/PR status. Multivariable analysis was performed among this weighted sample. Extended local therapy with anti-hormonal therapy still provided a survival advantage over other treatment cohorts (HR $=0.61,95 \% \mathrm{Cl}: 0.54-0.68)$. Anti-hormonal therapy had a significant impact upon survival for ER/PR positive tumors (HR = $1.45, \mathrm{p}<0.001)$ but not ER/PR negative tumors ( $H R=1.15, p=$ 0.188 ) (Table 5). Kaplan Meier analysis confirmed this survival advantage among these weighted cohorts with lumpectomy alone having $83.4 \%$ (95\% Cl: $80.7-85.7 \%$ ) 10 -year survival compared to $85.8 \%$ (95\% Cl: $84.7-86.9 \%)$ for extended local therapy and $88.5 \%$ (95\% Cl: $86.5-90.0 \%)$ for extended local plus anti-hormonal therapy $(p<0.001)$ (Figure 2$)$.

\section{Discussion}

Patients diagnosed with DCIS have an excellent prognosis with an estimated 20-year disease-specific mortality of $3 \%$ $[12,13]$. Given the excellent prognosis of DCIS, the optimal treatment to reduce mortality without added toxicity of additional treatment is controversial. Among this cohort of DCIS patients, additional local therapy beyond breast conserving surgery (BCS) alone with either radiation or unilateral mastectomy and the further addition of anti-hormonal therapy reduced mortality by $2.2 \%$ and $3.5 \%$ at 60 months and by $2.4 \%$ and $5.1 \%$ at 120 months, among matched cohorts respectively (see (Figure 2)).

Characteristics and treatment patterns of patients diagnosed with DCIS are shown in (Table 1), the demographics

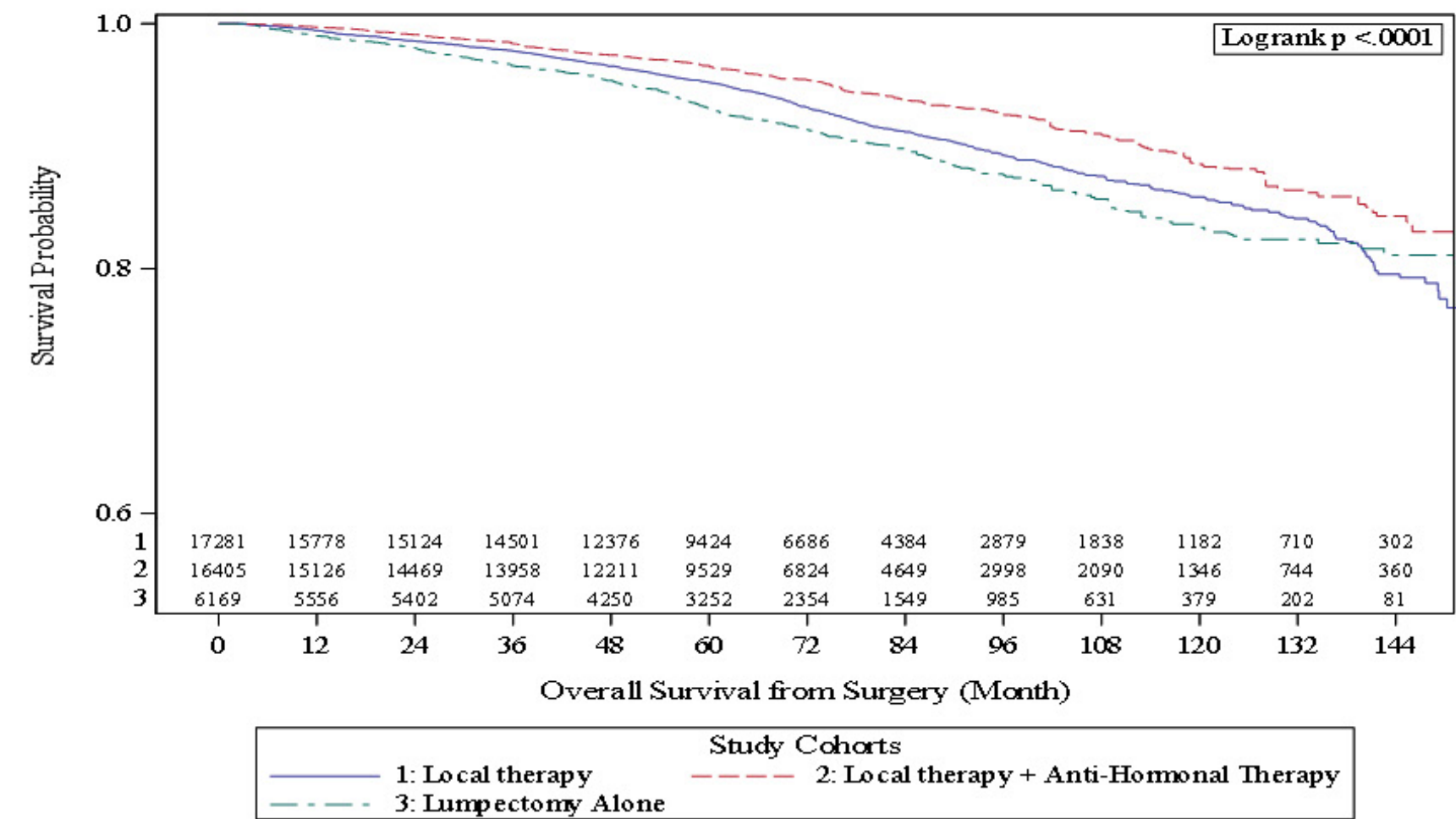

\begin{tabular}{|l|l|l|l|l|l|l|}
\hline Study Cohorts & No. of Subject & Event & Censored & $\begin{array}{l}\text { Median Survival } \\
\text { (95\% Cl) }\end{array}$ & $\begin{array}{l}\mathbf{6 0} \text { Mo } \\
\text { Survival }\end{array}$ & $\begin{array}{l}\mathbf{1 2 0} \text { Mo } \\
\text { Survival }\end{array}$ \\
\hline Local therapy & 15054 & $1063(7 \%)$ & $13991(93 \%)$ & $\begin{array}{l}\text { NA } \\
\text { (NA, NA) }\end{array}$ & $\begin{array}{l}95.2 \% \\
(94.8 \%, 95.6 \%)\end{array}$ & $(84.7 \%, 86.9 \%)$ \\
\hline $\begin{array}{l}\text { Local therapy }+ \\
\text { Anti-Hormonal } \\
\text { Therapy }\end{array}$ & 13989 & $580(4 \%)$ & $13409(96 \%)$ & NA & $96.5 \%$ & $88.5 \%$ \\
\hline $\begin{array}{l}\text { Lumpectomy } \\
\text { Alone }\end{array}$ & 4676 & $544(12 \%)$ & $4132(88 \%)$ & NA & $(96.0 \%, 96.9 \%)$ & $(86.9 \%, 90.0 \%)$ \\
\hline
\end{tabular}

\begin{tabular}{|l|l|l|}
\hline Study Cohorts & Overall Survival from Surgery (Month) & Survival Rate (95\% Cl) \\
\hline Local therapy & 120 & $85.8 \%(84.6 \%, 86.9 \%)$ \\
\hline Local therapy + Hormone & 120 & $88.3 \%(86.5 \%, 89.9 \%)$ \\
\hline Lumpectomy Alone & 120 & $83.3 \%(80.7 \%, 85.6 \%)$ \\
\hline
\end{tabular}

Figure 2: KM Overall Survival ATE SW. 
of our cohort are similar to other national studies [12]. Our results are similar to a recent SEER analysis: $14 \%$ of patients received lumpectomy alone, $44 \%$ received additional local therapy- $65 \%$ of whom received radiation and $35 \%$ of whom received unilateral mastectomy and $41 \%$ of patients received adjuvant anti-hormonal therapy. On multivariate analysis, demographic variables associated with decreased survival in our study included those previously reported in the literature including African-American race, increased Charlson-Deyo score, and Medicaid insurance [19-21]. Older patients had a decreased survival. Although younger patients have previously been shown to have decreased breast-cancer survival, in this cohort, older patients had decreased survival likely due to the NCDB's inability to differentiate overall and breast-cancer survival $[12,22,23]$. Tumor size $(>2 \mathrm{~cm})$ was associated with decreased survival on MVA. Positive margins were entirely excluded, margin size is not included in the NCDB. Tumor grade and histology and receptor status were not associated with survival on MVA. Although studies have attempted to define "low-risk" DCIS as low-intermediate grade, $<2.5 \mathrm{~cm}$ tumors with negative margins $(>1 \mathrm{~cm})$ which may be appropriate for less aggressive treatment, our results reflected the findings of ECOG-ACRIN E5194 that determined grade was nota useful marker for predicting risk of recurrence $[22,24]$. Our results demonstrate that papillary histology was associated with decreased survival on UVA but not MVA. In contrast, the SEER analysis of 108,196 patients performed by Narod etaldemon strated poor prognosis of comedocarcinoma histology [12]. Comedonecrosis is often associated with higher-grade tumors, which were not significantly associated with decreased survival in our study; in contrast, papillary histology has been shown to be more likely associated with invasive cancers and perhaps could represent missed invasive cancers among this cohort and a limitation of this and other NCDB studies. More recent efforts have examined the role of molecular markers in predicting the risk of recurrence after surgical excision [25-30]. ER/PR negative tumors were associated with decreased survival on UVA but not MVA among our cohort. Such tumors have been shown to be associated with decreased survival likely because they do not benefit from the addition of anti-hormonal therapy, although the use of anti-hormonal treatment has been inconsistent as demonstrated in this cohort $83 \%$ of patients were hormonal receptor positive but only $41 \%$ of anti-hormonal positive patients received anti-hormonal therapy [12]. Although not routinely reported, HER2 did not demonstrate an association with survival among our cohort, the clinical implications of HER2 among DCIS patients is in need of additional study [31].

Both univariate and multivariate analyses of our cohort demonstrated additional treatment beyond lumpectomy alone to be protective and additive. These results were confirmed on Kaplan-Meier analysis stratified by treatment type. Lumpectomy alone had the poorest OS (76.9\%) compared to additional local therapy (85.7\%) and additional local therapy plus anti-hormonal therapy $(90.5 \%)(p<0.001)$ (Figure 1). There is a multitude of conflicting evidence regarding the survival benefit of additional treatment beyond lumpectomy alone for DCIS such as radiation, mastectomy and/ or anti-hormonal therapy. Randomized trials have demon- strated reduced local recurrence among DCIS patients who receive these additional therapies but lack sufficient power to demonstrate a survival benefit [24,32-39]. Recent population-based studies have demonstrated conflicting results with some suggesting a survival benefit of radiotherapy among a certain subset of "high-risk" patients [13,14]. A recent SEER analysis by Narod of 108,196 patients and a subset analysis of 2947 patients performed by Giannakeas, et al. found that radiotherapy reduced recurrence without decreasing mortality, however, their analysis also demonstrated a subset of patients diagnosed with DCIS who subsequently died of invasive breast cancer without evidence of prior local recurrence, and therefore concluded DCIS has the potential for invasive and even systemic behavior and warrants consideration of systemic therapy to improve survival. The Narod and Giannakeas SEER analyses did not evaluate the impact of anti-hormonal therapy upon recurrence or survival among DCIS patients [13]. A 2018 prospective study of 9,938 women treated in the UK demonstrated a reduction in recurrence but not mortality among patients who used radiation and anti-hormonal therapy [40]. Given our cohort (34,444 patients) is much larger, and one of the larger cohort studies to report the effects of anti-hormonal therapy upon survival for DCIS, our study may have adeqeuate power to capture the small but potentially significant benefits of anti-hormonal therpay upon survival for patients diagnosed with DCIS.

Given the spectrum of treatment options available for the treatment of DCIS, three treatment groups of greatest interest were chosen for additional analysis and matching: Lumpectomy alone, extended local therapy (lumpectomy + XRT or mastectomy) and extended local therapy + anti-hormonal therapy. These three groups were chosen in order to re-examine in particular the mortality benefit of systemic therapy for DCIS patients. Associations between prognostic variables of interest and these three treatment groups were analyzed, as shown in (Table 3). Patients who received lumpectomy alone were older (mean age 63), more likely to have Medicare and have tumors with ductal or papillary histology, well-differentiated and smaller (mean tumor size $1.34 \mathrm{~cm}$ ).

Given the differences between these three treatment groups, a survival analysis was performed among cohorts via average treatment effect weighting (ATE). After matching cohorts, patients who received lumpectomy alone had a poorer survival (83.3\%) compared to the addition of extended local therapy (85.8\%) and anti-hormonal therapy (88.3\%) after 120 months $(p<0.001)$ (Figure 2). Furthermore, on Cox regression analysis, additional local therapy beyond lumpectomy alone was protective and the addition of anti-hormonal therapy added additional survival benefit to extended local therapy (Table 5). The survival benefit of anti-hormonal therapy was limited to patients with hormone receptor positive tumors suggesting that despite NCDB being unable to capture disease specific mortality, anti-hormonal therapy was potentially impacting survival among this cohort. Our results suggest that additional therapy to surgical excision of DCIS, both radiation and anti-hormonal, be considered to reduce both recurrence and mortality for patients diagnosed with DCIS.

Given the overall excellent survival of DCIS, prior studies 
have attempted to identify a subset of patients with an increased risk of mortality from breast cancer that may particularly benefit from additional therapy. Certain clinicopathologic factorsuch as higher nuclear grade, younger age, larger tumor size have been shown to be associated with reduced survival and prognostic scoring model shave been proposed to delineate which patients may safely be omitted from radiotherapy [23]. The survival benefit of additional therapy among our ATE-weighted cohorts questions such conclusions and practices and warrants additional investigation as current prospective studies have failed to clearly identify a subset of patients who can safely omit radiotherapy and/or anti-hormonal therapy following lumpectomy [14,24,41,42].

Our study is retrospective as thus unable to determine causality for the associations we have observed. The NCDB, like other cancer registries, does not capture recurrence or disease-specific mortality, and therefore, we are unable to assess the true breast-cancer specific mortality among this cohort, however, it is the largest registry available for study of a disease that requires ample power for mortality analysis. We have attempted to utilize ATE-weighting to minimize bias and assess mortality differences between matched treatment cohorts. Given the NCDB's large national data source, our results are generalizable to patients across the US. Further investigation is warranted to confirm the effects of additional therapies upon mortality for DCIS, and to attempt to identify subsets of patients who may benefit or may safely be omitted from additional therapy for DCIS.

\section{Conclusion}

Among patients diagnosed with DCIS cases diagnosed between 2004-2015, lumpectomy alone (14\%) was the sole treatment for one in seven cases. However, using ATE-weighted methodology to compare outcomes of patients diagnosed with DCIS, we observed significantly better overall survival was observed with the addition of extended local therapy (HR $=0.81,95 \% \mathrm{Cl}: 0.73-0.90)$ and anti-hormonal therapy $(\mathrm{HR}=$ $0.61,95 \% \mathrm{Cl}: 0.54-0.68)$ compared to those who had lumpectomy alone $(p<0.001)$. Although the NCDB does not capture recurrence or breast-cancer specific mortality, these results suggest that additional therapy beyond surgery alone may improve survival for patients diagnosed with DCIS. Further investigation is indicated to elucidate the mechanisms of DCIS invasion and to identify the subset of patients for whom additional therapy may be beneficial or safely omitted.

\section{Acknowledgement}

Research reported in this publication was supported in part by the Biostatistics and Bioinformatics Shared Resource of Winship Cancer Institute of Emory University and $\mathrm{NIH} / \mathrm{NCl}$ under award number P30CA138292. The content is solely the responsibility of the authors and does not necessarily represent the official views of the National Institutes of Health. The data used in the study are derived from a de-identified NCDB file. The American College of Surgeons and the Commission on Cancer have not verified and are not responsible for the analytic or statistical methodology employed, or the conclusions drawn from these data by the investigator.

\section{References}

1. Allegra CJ, Aberle DR, Ganschow P, et al. (2010) National institutes of health state-of-the-science conference statement: Diagnosis and management of ductal carcinoma in situ September 22-24, 2009. J Natl Cancer Inst 102: 161-169.

2. Burstein HJ, Polyak K, Wong JS, et al. (2004) Ductal carcinoma in situ of the breast. N Engl J Med 350: 1430-1441.

3. Zujewski JA, Harlan LC, Morrell DM, et al. (2011) Ductal carcinoma in situ: Trends in treatment over time in the US. Breast Cancer Res Treat 127: 251-257.

4. Schwartz GF, Solin LJ, Olivotto IA, et al. (2000) Consensus conference on the treatment of in situ ductal carcinoma of the breast, April 22-25, 1999. Cancer 88: 946-954.

5. Dodwell D, Clements K, Lawrence G, et al. (2007) Radiotherapy following breast-conserving surgery for screen-detected ductal carcinoma in situ: Indications and utilisation in the UK. Interim findings from the Sloane Project. Br J Cancer 97: 725-729.

6. Baxter NN, Virnig BA, Durham SB, et al. (2004) Trends in the treatment of ductal carcinoma in situ of the breast. J Natl Cancer Inst 96: 443-448.

7. Smith GL, Smith BD, Haffty BG (2006) Rationalization and regionalization of treatment for ductal carcinoma in situ of the breast. Int J Radiat Oncol Biol Phys 65: 1397-1403.

8. Sakorafas GH, Farley DR, Peros G (2008) Recent advances and current controversies in the management of DCIS of the breast. Cancer Treat Rev 34: 483-497.

9. Levinsohn E, Altman M, Chagpar AB (2018) Controversies Regarding the Diagnosis and Management of Ductal Carcinoma In Situ. Am Surg 84: 1-6.

10. Buchholz TA, Haffty BG, Harris JR (2007) Should all patients undergoing breast conserving therapy for DCIS receive radiation therapy? Yes. Radiation therapy, an important component of breast conserving treatment for patients with ductal carcinoma in situ of the breast. J Surg Oncol 95: 610-613.

11. Silverstein MJ, Lagios MD (2007) Should all patients undergoing breast conserving therapy for DCIS receive radiation therapy? No. One size does not fit all: An Argument against the routine use of radiation therapy for all patients with ductal carcinoma in situ of the breast who elect breast conservation. J Surg Oncol 95: 605-609.

12. Narod SA, Iqbal J, Giannakeas V, et al. (2015) Breast cancer mortality after a diagnosis of ductal carcinoma in Situ. JAMA Oncol 1: 888-896.

13. Giannakeas V, Sopik V, Narod SA (2018) A comparison of two models for breast cancer mortality for women with ductal carcinoma in situ: an SEER-based analysis. Breast Cancer Res Treat 169: 587-594.

14. Sagara Y, Freedman RA, Vaz-Luis I, et al. (2016) Patient prognostic score and associations with survival improvement offered by radiotherapy after breast-conserving surgery for ductal carcinoma in Situ: A population-based longitudinal cohort study. J Clin Oncol 34: 1190-1196.

15. Grimm LJ, Shelley Hwang E (2016) Active surveillance for DCIS: The importance of selection criteria and monitoring. Ann Surg Oncol 23: 4134-4136.

16. Winchester DP, Stewart AK, Bura C, et al. (2004) The national cancer data base: A clinical surveillance and quality improvement tool. J Surg Oncol 85: 1-3. 
17. Austin PC (2009) Balance diagnostics for comparing the distribution of baseline covariates between treatment groups in propensity-score matched samples. Stat Med 28: 3083-3107.

18. Liu Y, Nickleach DC, Zhang C, et al. (2018) Carrying out streamlined routine data analyses with reports for observational studies: Introduction to a series of generic SAS macros. F1000Res 7: 1955.

19. Bleicher RJ, Ruth K, Sigurdson ER, et al. (2016) Time to surgery and breast cancer survival in the United States. JAMA Oncol 2: 330-339.

20. Shi R, Diaz R, Shi Z, (2016) The effect of payer status on survival of patients with stage $\mathrm{I} / \mathrm{II}$ non-small cell lung cancer: NCDB 19982011. Anticancer Res 36: 319-326.

21. Chen AY, Halpern M (2007) Factors predictive of survival in advanced laryngeal cancer. Arch Otolaryngol Head Neck Surg 133: $1270-1276$.

22. Rosenberg J, Chia YL, Plevritis S (2005) The effect of age, race, tumor size, tumor grade, and disease stage on invasive ductal breast cancer survival in the U.S. SEER database. Breast Cancer Res Treat 89: 47-54.

23. Ayanian JZ, Kohler BA, Abe T, et al. (1993) The relation between health insurance coverage and clinical outcomes among women with breast cancer. N Engl J Med 329: 326-331.

24. McCormick B, Winter K, Hudis C, et al. (2015) RTOG 9804: A Prospective randomized trial for good-risk ductal carcinoma in situ comparing radiotherapy with observation. J Clin Oncol 33: 709715.

25. Solin LJ, Gray R, Baehner FL, et al. (2013) A multigene expression assay to predict local recurrence risk for ductal carcinoma in situ of the breast. J Natl Cancer Inst 105: 701-710.

26. Rakovitch E, Nofech-Mozes S, Hanna W, et al. (2015) A population-based validation study of the DCIS Score predicting recurrence risk in individuals treated by breast-conserving surgery alone. Breast Cancer Res Treat 152: 389-398.

27. Kerlikowske K, Molinaro AM, Gauthier ML, et al. (2010) Biomarker expression and risk of subsequent tumors after initial ductal carcinoma in situ diagnosis. J Natl Cancer Inst 102: 627-637.

28. Rakovitch E, Nofech-Mozes S, Hanna W, et al. (2012) HER2/neu and $\mathrm{Ki}-67$ expression predict non-invasive recurrence following breast-conserving therapy for ductal carcinoma in situ. Br J Cancer 106: 1160-1165.

29. Williams KE, Barnes NL, Cramer A, et al. (2015) Molecular phenotypes of DCIS predict overall and invasive recurrence. Ann Oncol 26: 1019-1025.

30. Pal SK, Lau SK, Kruper L, et al. (2010) Papillary carcinoma of the breast: An overview. Breast Cancer Res Treat 122: 637-645.

31. Siziopikou KP, Anderson SJ, Cobleigh MA, et al. (2013) Prelimi- nary results of centralized HER2 testing in ductal carcinoma in situ (DCIS): NSABP B-43. Breast Cancer Res Treat 142: 415-421.

32. Fisher B, Dignam J, Wolmark N, et al. (1999) Tamoxifen in treatment of intraductal breast cancer: National Surgical Adjuvant Breast and Bowel Project B-24 randomised controlled trial. Lancet 53: 1993-2000.

33. Fisher B, Dignam J, Wolmark N, et al. (1998) Lumpectomy and radiation therapy for the treatment of intraductal breast cancer: Findings from National Surgical Adjuvant Breast and Bowel Project B-17. J Clin Oncol 16: 441-452.

34. Fisher B, Land S, Mamounas E, et al. (2001) Prevention of invasive breast cancer in women with ductal carcinoma in situ: An Update of the National Surgical Adjuvant Breast and Bowel Project experience. Semin Oncol 28: 400-418.

35. Wapnir IL, Dignam JJ, Fisher B, et al. (2011) Long-term outcomes of invasive ipsilateral breast tumor recurrences after lumpectomy in NSABP B-17 and B-24 randomized clinical trials for DCIS. J Natl Cancer Inst 103: 478-488.

36. Donker M, Litiere S, Werutsky G, et al. (2013) Breast-conserving treatment with or without radiotherapy in ductal carcinoma In Situ: 15-year recurrence rates and outcome after a recurrence, from the EORTC 10853 randomized phase III trial. J Clin Oncol 31: 4054-4059.

37. Bijker N, Peterse JL, Duchateau L, et al. (2001) Risk factors for recurrence and metastasis after breast-conserving therapy for ductal carcinoma-in-situ: analysis of European Organization for Research and Treatment of Cancer Trial 10853. J Clin Oncol 19: 2263-2271.

38. Cuzick J, Sestak I, Pinder SE, et al. (2010) Effect of tamoxifen and radiotherapy in women with locally excised ductal carcinoma in situ: long-term results from the UK/ANZ DCIS trial. Lancet Oncol 12: 21-29.

39. Warnberg F, Garmo H, Emdin S, et al. (2014) Effect of radiotherapy after breast-conserving surgery for ductal carcinoma in situ: 20 years follow-up in the randomized SweDCIS Trial. J Clin Oncol 32: 3613-3618.

40. Thompson AM, Clements K, Cheung S, et al. (2018) Management and 5-year outcomes in 9938 women with screen-detected ductal carcinoma in situ: the UK Sloane Project. Eur J Cancer 101: 210-219.

41. Hughes LL, Wang M, Page DL, et al. (2009) Local excision alone without irradiation for ductal carcinoma in situ of the breast: A Trial of the Eastern Cooperative Oncology Group. J Clin Oncol 27: 5319-5324.

42. Solin LJ, Gray R, Hughes LL, et al. (2015) Surgical Excision Without Radiation for Ductal Carcinoma in Situ of the Breast: 12-Year Results From the ECOG-ACRIN E5194 Study. J Clin Oncol 33: 39383944.

DOI: $10.36959 / 739 / 526$

Copyright: (c) 2020 Broecker JS, et al. This is an open-access article distributed under the terms of the Creative Commons Attribution License, which permits unrestricted use, distribution, and reproduction in any medium, provided the original author and source are credited. 